\title{
Perspective Progress on the Study and Treatment of Depressive Illness
}

\author{
William A Carlezon Jr*,' and Elena Chartoff' \\ 'Behavioral Genetics Laboratory, Department of Psychiatry, Harvard Medical School, McLean Hospital, Belmont, MA, USA
}

Neuropsychopharmacology (2009) 34, I36 I-1362; doi:10.1038/npp.2009.16; published online 25 February 2009

Neuropsychopharmacology was first published 21 years ago. The early issues are filled with work from past, present, and future thought leaders in the field. The similarities between the articles that were published in 1988 and those being published today are striking. This is a testament to the foresight of those researchers who were the first to work at the interface of the central nervous system (neuro), the mind (psycho), and drugs (pharmacology).

There was great emphasis on schizophrenia in the earliest issues of the journal. At the time, it was becoming clear that new drugs (eg, clozapine) had major advantages over classic drugs (eg, haloperidol). There was enthusiasm for the idea that the increased safety and efficacy of drugs such as clozapine had much to teach us, and that an improved understanding of how these drugs worked would enable new insights into the pathophysiology of the disorder (Meltzer, 1988). In parallel, advances in molecular biology and molecular pharmacology offered an improved ability to identify and catalog the many receptor targets of psychiatric medications, as well as the opportunity to use multidisciplinary approaches to tease out the substrates of desirable and undesirable drug effects.

The early pioneers in neuropsychopharmacology blazed a trail that we continue to follow today. A primary strategy in our field is 'drug-centric': we study how psychotropic medications work, we look for common actions among drugs that are efficacious in the treatment of a particular disorder, and we use this information to develop working hypotheses about pathophysiology. This strategy continues to hold promise for developing new and improved medications. The cellular targets under investigation have become extraordinarily complex and multidisciplinary, reflecting a continued trend toward blending fields and perspectives that have traditionally been distinct. In this issue, Zhou et al (2009) report that mood stabilizers have common intracellular effects on microRNAs, singlestranded RNA molecules that regulate gene function. This is an insightful and innovative study of standard psychiatric

*Correspondence: Dr WA Carlezon, Department of Psychiatry, Harvard Medical School, McLean Hospital, MRC 217, I 15 Mill Street, Belmont, MA 02478, USA, Tel: + 617855 2021, Fax: + 617855 2023, E-mail: bcarlezon@mclean.harvard.edu

Received 13 January 2009; accepted 13 January 2009 drugs in combination with targets and concepts often associated with cancer research. The approaches used in this report have the potential to identify new targets for psychiatric drugs while simultaneously increasing our understanding of cell function, producing a breadth of knowledge applicable to a variety of fields.

Reports from the earliest issues of Neuropsychopharmacology also alluded to the need for improved models of psychiatric disorders, including major depression (Simson and Weiss, 1988). The models and screening procedures used today are virtually identical to those being used in the 1980s; there have been few advances. It is remarkable that those of us engaged in preclinical depression research cannot easily distinguish a molecule that would revolutionize the treatment of depressive illness from one that is no better than those available now. Of even greater concern is the realization that screening techniques that have evolved to identify drugs with certain pharmacological effects might not be sensitive to new, mechanistically distinct agents. These issues highlight the need to combine 'drug-centric' approaches with 'brain-centric' approaches. Again, taking clues from strategies used in other disciplines might be helpful. For example, it might be misleading to extrapolate the causes of cancer from an understanding of how traditional chemotherapies treat it. A complementary approach is to focus on understanding normal function, what disrupts it, and how to fix what is broken. Designing such studies in psychiatry is a daunting task because the brain is a particularly complicated assortment of cells and circuits. Perhaps more importantly, at present we do not have models that we trust to tell us when we have triggered the signs and symptoms of psychiatric illness in laboratory animals.

In this issue, there are several reports that take a 'braincentric' approach by using ethologically relevant paradigms to model depressive illness. It has been established that stressful events increase vulnerability to depressive disorders in people (Keller et al, 2007). Law et al (2009) use a powerful form of stress (early parental deprivation in marmosets) to understand how the brain changes in response to environmental factors that trigger signs of depression. Importantly, they describe molecular adaptations that are, and are not, associated with depressive behavior. Similarly, Surget et al (2009) use a regimen of chronic exposure to stress to cause depressive-like signs in 
mice. They report that antidepressant drugs have substantially different molecular consequences in mice that are normal and those showing depressive-like behavior, highlighting the growing realization in the field that the brains of individuals with psychiatric illness might react in different (and sometimes paradoxical) ways to psychotropic drugs. They also find that the molecular signatures of chronic stress and antidepressant treatments are brain region-specific, which underscores the importance of understanding neural circuitry and not just pharmacological targets.

Surget et al (2009) implicate brain receptors for the stress-related peptide corticotrophin-releasing factor (CRF) in the development and relief of depressive symptoms in their models. Todorovic et al (2009) come to similar conclusions, and go on to mimic some of the physiological consequences of stress by using genetic techniques to elevate CRF activity in the brain. They provide elegant detail on the intracellular mechanisms by which stress might trigger depressive-like states, providing an example of 'brain-centric' research that identifies a potential molecular target for the development of novel antidepressant drugs. Bosch et al (2009) take advantage of the monogamous nature of prairie voles to show that CRF receptors play key roles in social bonding and the pathological consequences of losing a mate, both of which clearly influence mental health in humans.

The potential suitability of prairie voles for studies of complex human emotions, such as grief, might represent a modern-day example of a 1920s-era idea now known as the Krogh principle: 'for many problems there is an animal on which it can be most conveniently studied' (Krebs, 1975). Developing trustworthy tests in rats, mice, or voles that reflect the core symptoms of psychiatric illness is a top priority because genetic engineering provides us with the unprecedented opportunity to create entirely new animals on which a variety of problems in neuropsychopharmacology can be most conveniently - or insightfully - studied using 'brain-centric' approaches.

\section{ACKNOWLEDGEMENTS}

We were supported in part by grants from the National Institutes of Health (MH063266 and DA012736 to WAC; DA023606 to EHC) while writing this commentary.

\section{DISCLOSURE}

We report no conflicts of interest related to this commentary. Dr Carlezon has a US patent and several US patent applications covering the use of novel approaches for treating depressive illness. He has received compensation from Infinity Pharmaceuticals, Myneurolab.com, and Psychogenics. Dr Chartoff has nothing to disclose.

\section{REFERENCES}

Bosch OJ, Nair HP, Ahern TH, Neumann ID, Young LJ (2009). The CRF system mediates increased passive stress-coping behavior following the loss of a bonded partner in a monogamous rodent. Neuropsychopharmacol, in press.

Keller MC, Neale MC, Kendler KS (2007). Association of different adverse life events with distinct patterns of depressive symptoms. Am J Psychiatry 164: 1521-1529.

Krebs HA (1975). The August Krogh principle: 'For many problems there is an animal on which it can be most conveniently studied. J Exp Zoology 194: 221-226.

Law AJ, Pei Q, Walker M, Gordon-Andrews H, Weickert CS, Feldon J et al (2009). Early parental deprivation in the marmoset monkey produces long-term changes in hippocampal expression of genes involved in synaptic plasticity and implicated in mood disorder. Neuropsychopharmacol, in press.

Meltzer HY (1988). New insights into schizophrenia through atypical antipsychotic drugs. Neuropsychopharmacol 1: 193-196.

Surget A, Wang Y, Leman S, Ibarguen-Vargas Y, Edgar N, Griebel $\mathrm{G}$ et al (2009). Corticolimbic transcriptome changes are statedependent and region-specific in a rodent model of depression and of antidepressant reversal. Neuropsychopharmacol, in press.

Simson PE, Weiss JM (1988). Altered activity of the locus coeruleus in an animal model of depression. Neuropsychopharmacol 1: 287-295.

Todorovic C, Sherrin T, Pitts M, Hippel C, Rayner M, Spiess J (2009). Suppression of the MEK/ERK signaling pathway reverses depression-like behaviors of $\mathrm{CRF}(2)$-deficient mice. Neuropsychopharmacol, in press.

Zhou R, Yuan P, Wang Y, Hunsberger JG, Elkahloun A, Wei Y et al (2009). Evidence for selective microRNAs and their effectors as common long-term targets for the actions of mood stabilizers. Neuropsychopharmacol, in press. 